\title{
POLYNOMIAL EQUIVALENCE OF FINITE RINGS
}

\author{
GEORG GRASEGGER, GÁBOR HORVÁTH, AND KEITH A. KEARNES
}

\begin{abstract}
We prove that $\mathbb{Z}_{p^{n}}$ and $\mathbb{Z}_{p}[t] /\left(t^{n}\right)$ are polynomially equivalent if and only if $n \leq 2$ or $p^{n}=8$. For the proof, employing Bernoulli numbers, we provide the polynomials which compute the carry-on part for the addition and multiplication in base $p$. As a corollary, we characterize finite rings of $p^{2}$ elements up to polynomial equivalence.
\end{abstract}

\section{INTRODUCTION}

One of the most basic objectives of algebra is to characterize different algebraic structures up to certain equivalences. Very often, characterization up to isomorphism is considered. In this paper, we investigate certain finite rings, and characterize them up to polynomial equivalence.

A polynomial function over an algebra is a function built up from projections, constant functions and basic operations using composition. Two algebras are polynomially equivalent if they are defined on the same domain and have the same polynomial functions [5]. It is easy to see that two algebras are polynomially equivalent if and only if the basic operations of one algebra can be expressed as polynomials of the other algebra, and vice versa.

The question to characterize algebras up to polynomial equivalence arises quite naturally. From a Computer Science perspective, polynomials capture the functions computable by the algebra, and polynomial equivalent algebras can compute exactly the same functions. In many cases though, different algebras are not defined on the same domain, but can still be polynomial equivalent if the elements are identified via some bijection $\varphi$. Therefore in the paper we use the following definition of polynomial equivalence.

Date: September 8, 2012.

2010 Mathematics Subject Classification. 13M10 (13B25, 11B68).

Key words and phrases. polynomial equivalence, finite rings, Bernoulli numbers, carry for base $p$ addition, carry for base $p$ multiplication.

Corresponding author: Gábor Horváth (ghorvath@science.unideb.hu).

The first author was partially supported by the strategic program "Innovatives OÖ 2010plus" by the Upper Austrian Government and by the Austrian Science Fund (FWF): W1214-N15, project DK11. The second author was partially supported by the Austrian Science Fund (FWF): P24077 and by the János Bolyai Research Scholarship of the Hungarian Academy of Sciences. 
Definition 1. Let $\mathcal{R}_{1}=\left(R_{1},+_{1}, \times_{1}\right)$ and $\mathcal{R}_{2}=\left(R_{2},+_{2}, \times_{2}\right)$ be two rings, and let $\varphi: R_{1} \rightarrow R_{2}$ be a bijection. We say that the rings $\mathcal{R}_{1}$ and $\mathcal{R}_{2}$ are polynomially equivalent via $\varphi$ if there exist polynomial functions $f_{1}, g_{1}$ over $\mathcal{R}_{1}$ and polynomial functions $f_{2}, g_{2}$ over $\mathcal{R}_{2}$ such that for arbitrary $x, y \in R_{1}$ we have

$$
\begin{aligned}
\varphi\left(x+{ }_{1} y\right) & =f_{2}(\varphi(x), \varphi(y)), \\
\varphi\left(x \times_{1} y\right) & =g_{2}(\varphi(x), \varphi(y)), \\
\varphi\left(f_{1}(x, y)\right) & =\varphi(x)+{ }_{2} \varphi(y), \\
\varphi\left(g_{1}(x, y)\right) & =\varphi(x) \times_{2} \varphi(y) .
\end{aligned}
$$

In particular, $\mathcal{R}_{1} \stackrel{\varphi}{\simeq}\left(R_{2}, f_{2}, g_{2}\right)$ and $\left(R_{1}, f_{1}, g_{1}\right) \stackrel{\varphi}{\simeq} \mathcal{R}_{2}$. One can extend this notion to arbitrary algebras in a natural way, but we skip the general definition as the scope of the paper is limited to rings. Note, however, that if the elements of the two rings are identified via the bijection $\varphi$, then our definition for polynomial equivalence coincides with the usual one.

One of the most interesting cases of nonisomorphic algebras that are polynomially equivalent comes from group theory. Any two nonabelian simple groups are polynomially complete by [9], thus their polynomial equivalence type is determined by their order. There are nonisomorphic nonabelian simple groups of the same order, e.g. both $P S L(4,2)$ and $P S L(3,4)$ have 20160 elements [11]. Similarly, any two simple unital rings of the same order are polynomially equivalent [8] but not necessarily isomorphic. In particular, $M_{m}(q)$ is isomorphic to $M_{n}(r)$ if and only if $m=n$ and $q=r$, but they are polynomially equivalent if and only if $q^{m^{2}}=r^{n^{2}}$.

In our paper we consider the rings $\mathbb{Z}_{p^{n}}$ and $\mathbb{Z}_{p}[t] /\left(t^{n}\right)$ for positive integers $n$ and primes $p$. These rings are isomorphic only for $n=1$, but always have the same number of elements, same number of unary polynomial functions [4] and the same ideal structure. Their elements even have a natural correspondence: for $P=\{0,1, \ldots, p-1\}$, every element of $\mathbb{Z}_{p^{n}}$ can be uniquely written in the form of $\sum_{i=0}^{n-1} a_{i} p^{i}\left(a_{i} \in\right.$ $P)$, and every element of $\mathbb{Z}_{p}[t] /\left(t^{n}\right)$ can be uniquely written in the form of $\sum_{i=0}^{n-1} a_{i} t^{i}\left(a_{i} \in P\right)$. We determine when these two rings are polynomially equivalent via some bijection.

Theorem 2. Let $p$ be a positive prime and $n$ a positive integer. Let $P=\{0,1, \ldots, p-1\}$. The two rings $\mathbb{Z}_{p^{n}}$ and $\mathbb{Z}_{p}[t] /\left(t^{n}\right)$ are

(1) polynomially equivalent via $\varphi: \mathbb{Z}_{p^{n}} \rightarrow \mathbb{Z}_{p}[t] /\left(t^{n}\right), \sum_{i=0}^{n-1} a_{i} p^{i} \mapsto$ $\sum_{i=0}^{n-1} a_{i} t^{i}\left(a_{i} \in P\right)$ for $n \leq 2$ and for $p^{n}=8$;

(2) not polynomially equivalent via any bijection $\mathbb{Z}_{p^{n}} \rightarrow \mathbb{Z}_{p}[t] /\left(t^{n}\right)$ if $n \geq 3$, except for $p^{n}=8$.

The proof of Theorem 2 consists of two main parts. We prove item (1) in Section 3 and item (2) in Section 4. For proving item (1) we give the 
polynomials for $f_{1}, g_{1}, f_{2}, g_{2}$. The main differences between the addition and multiplication of the two rings $\mathbb{Z}_{p^{2}}$ and $\mathbb{Z}_{p}[t] /\left(t^{2}\right)$ are essentially the carry-on parts of addition and multiplication in base $p$. It turns out that to define $f_{1}, g_{1}, f_{2}, g_{2}$, one has to determine the polynomials for these carry-on functions. Note, that the existence of such polynomials follows from the fact that $\mathbb{Z}_{p}$ is polynomially complete, hence every function can be represented as a polynomial (see e.g. [10]). Moreover, the polynomial equivalence of $\mathbb{Z}_{p^{2}}$ and $\mathbb{Z}_{p}[t] /\left(t^{2}\right)$ follows from the results of $[1,6]$, in particular the existence of $f_{1}$ and $f_{2}$ is proved in [1, Lemma 22]. We, in fact, provide the polynomials expressing the carry-on part of the addition and multiplication in base $p$. We introduce these polynomials employing Bernoulli numbers, then prove the addition part of item (1) in Section 3.1 and the multiplication part of item (1) in Section 3.2.

All the required notions and lemmas for the proof are summarized in Section 2. Finally, in Section 5 we apply Theorem 2 to characterize the rings containing $p^{2}$ elements up to polynomial equivalence and prove the following.

Corollary 3. Let us use the notations of [3] for rings having $p^{2}$ elements, that is let $A=\mathbb{Z}_{p^{2}}, B=p \mathbb{Z}_{p^{3}}, C=p^{2} \mathbb{Z}_{p^{4}}, D=\mathbb{Z}_{p} \oplus \mathbb{Z}_{p}, E$ and $F$ be the two noncommutative $p^{2}$-element rings, $G=\mathbb{Z}_{p}[t] /\left(t^{2}\right)$, $H=\mathbb{Z}_{p} \oplus p \mathbb{Z}_{p^{2}}, I=t \mathbb{Z}_{p}[t] /\left(t^{3}\right), J=p \mathbb{Z}_{p^{2}} \oplus p \mathbb{Z}_{p^{2}}$, and $K$ be the $p^{2}$-element field, where $\oplus$ denotes the direct sum of rings. Then $A$ is polynomially equivalent to $G, B$ is polynomially equivalent to $I$ for $p=2, E$ is polynomially equivalent to $F$, and no other two rings having $p^{2}$ elements are polynomially equivalent.

\section{Preliminaries}

2.1. Notations. Throughout the paper, $p$ always denotes a positive prime, $n$ a positive integer, and $m$ a nonnegative integer. We use $i$ and $j$ for running indices, $k$ for indexing Bernoulli numbers. We use $\equiv_{p}$ for indicating that the two sides are congruent modulo $p$. By $P$ we denote the set $\{0,1, \ldots, p-1\}$.

For rings $\mathcal{R}_{1}$ and $\mathcal{R}_{2}$, a function from $\mathcal{R}_{i}$ will be denoted by using the index $i \in\{1,2\}$. We denote the addition of $\mathcal{R}_{i}$ by $+_{i}$, the subtraction of $\mathcal{R}_{i}$ by $-_{i}$, and the multiplication of $\mathcal{R}_{i}$ by $\times_{i}(i \in\{1,2\})$. We write $+_{p}$ and $\times_{p}$ for the modulo $p$ addition and multiplication over $P$. Finally, we use the usual + and $\cdot$ for the usual addition and multiplication over the integers, unless we explicitly indicate otherwise.

2.2. Polynomially equivalent rings via a bijection. Let $\mathcal{R}_{1}$ and $\mathcal{R}_{2}$ be two finite rings having the same number of elements. Let $\varphi: \mathcal{R}_{1} \rightarrow \mathcal{R}_{2}$ be a bijection and assume $\mathcal{R}_{1}$ and $\mathcal{R}_{2}$ are polynomially equivalent via $\varphi$. Conjugating every polynomial function over $\mathcal{R}_{2}$ by adding $\varphi\left(0_{1}\right)$ to them, we can assume that $\varphi\left(0_{1}\right)=0_{2}$. 
Lemma 4. Let $\mathcal{R}_{1}$ and $\mathcal{R}_{2}$ be finite rings, $\varphi: \mathcal{R}_{1} \rightarrow \mathcal{R}_{2}$ a bijection. Assume $\mathcal{R}_{1}$ and $\mathcal{R}_{2}$ are polynomially equivalent via $\varphi$. Then there exists a bijection $\varphi^{\prime}: \mathcal{R}_{1} \rightarrow \mathcal{R}_{2}$ such that $\varphi^{\prime}\left(0_{1}\right)=0_{2}$, and $\mathcal{R}_{1}$ and $\mathcal{R}_{2}$ are polynomially equivalent via $\varphi^{\prime}$. Moreover, if $\mathcal{I}_{1} \triangleleft \mathcal{R}_{1}$, then $\varphi^{\prime}\left(\mathcal{I}_{1}\right) \triangleleft \mathcal{R}_{2}$.

Proof. Let $h_{2}$ be an arbitrary invertible unary polynomial over $\mathcal{R}_{2}$ such that $h_{2}\left(0_{2}\right)=\varphi\left(0_{1}\right)$. Such a polynomial exists, e.g. $h_{2}(x)=$ $x+{ }_{2} \varphi\left(0_{1}\right)$ suffices. Denote the inverse polynomial of $h_{2}$ by $h_{2}^{-1}$. Let $h_{1}: \mathcal{R}_{1} \rightarrow \mathcal{R}_{1}$ be the corresponding polynomial over $\mathcal{R}_{1}$, that is $\varphi\left(h_{1}(x)\right)=h_{2}(\varphi(x))$. Let $h_{1}^{-1}$ denote the inverse of $h_{1}$. Then for

$$
\begin{array}{rlrl}
\varphi^{\prime}: \mathcal{R}_{1} & \rightarrow \mathcal{R}_{2}, & x & \mapsto h_{2}^{-1}(\varphi(x)), \\
f_{1}^{\prime}: \mathcal{R}_{1} \times \mathcal{R}_{1} \rightarrow \mathcal{R}_{1}, & (x, y) & \mapsto h_{1}\left(f_{1}\left(h_{1}^{-1}(x), h_{1}^{-1}(y)\right)\right), \\
g_{1}^{\prime}: \mathcal{R}_{1} \times \mathcal{R}_{1} \rightarrow \mathcal{R}_{1}, & (x, y) & \mapsto h_{1}\left(g_{1}\left(h_{1}^{-1}(x), h_{1}^{-1}(y)\right)\right), \\
f_{2}^{\prime}: \mathcal{R}_{2} \times \mathcal{R}_{2} \rightarrow \mathcal{R}_{2}, & (x, y) & \mapsto h_{2}^{-1}\left(f_{2}\left(h_{2}(x), h_{2}(y)\right)\right), \\
g_{2}^{\prime}: \mathcal{R}_{2} \times \mathcal{R}_{2} \rightarrow \mathcal{R}_{2}, & (x, y) & \mapsto h_{2}^{-1}\left(g_{2}\left(h_{2}(x), h_{2}(y)\right)\right),
\end{array}
$$

the rings $\mathcal{R}_{1}$ and $\mathcal{R}_{2}$ are polynomially equivalent via $\varphi^{\prime}$, where $f_{1}^{\prime}, g_{1}^{\prime}$ correspond to $+_{2}$ and $\times_{2}$, and $f_{2}^{\prime}, g_{2}^{\prime}$ correspond to $+_{1}$ and $\times_{1}$, moreover $\varphi^{\prime}\left(0_{1}\right)=0_{2}$.

If $\mathcal{I}_{1} \triangleleft \mathcal{R}_{1}$ is an ideal, then the congruence defined by $\mathcal{I}_{1}$ is preserved by polynomials of $\mathcal{R}_{1}$, in particular is preserved by $f_{1}^{\prime}$ and $g_{1}^{\prime}$. Therefore the $\varphi^{\prime}$-image of this congruence is preserved by $+_{2}$ and $\times_{2}$, and thus is a congruence of $\mathcal{R}_{2}$. As every congruence of a ring is defined by an ideal and $\varphi^{\prime}\left(\mathcal{I}_{1}\right) \ni \varphi^{\prime}\left(0_{1}\right)=0_{2}, \varphi^{\prime}\left(\mathcal{I}_{1}\right)$ must be an ideal of $\mathcal{R}_{2}$.

Note, that a similar proof shows that for general algebras the $\varphi$-image of a coset of a congruence has to be a coset of a congruence.

2.3. Bernoulli Numbers. Bernoulli numbers are defined by the recurrence formula

$$
\sum_{k=0}^{m}\left(\begin{array}{c}
m+1 \\
k
\end{array}\right) B_{k}=0
$$

for $m \geq 1$, and $B_{0}=1[7$, Chapter 15$]$. With this definition we have $B_{1}=-1 / 2$, and $B_{k}=0$ for every other odd $k$.

A basic property of Bernoulli numbers [7, Chapter 15, Theorem 1] is that for all $m \geq 0, y \geq 1$ integers, we have

$$
\frac{1}{m+1} \sum_{k=0}^{m}(-1)^{k}\left(\begin{array}{c}
m+1 \\
k
\end{array}\right) B_{k} y^{m+1-k}=\sum_{k=1}^{y} k^{m} .
$$

For $2 \mid k$ Clausen [2] and Von Staudt [12] proved the following on the denominators of the Bernoulli numbers:

$$
B_{k}+\sum_{\substack{q \text { prime } \\(q-1) \mid k}} \frac{1}{q} \in \mathbb{Z}
$$


In particular, $B_{0}=1$ is an integer, and if $p-1>k \geq 1$, then $p$ does not divide the denominator of $B_{k}$ (in its simplified form). Thus $B_{k}$ for $p-1>k$ can be calculated modulo $p$. Furthermore we use a consequence of Voronoi's Congruence [7, Proposition 15.2.3], which relates the numerator and denominator of a Bernoulli number modulo an integer. Let $p \geq 3$ be a prime and $x$ an integer not divisible by $p$. For positive even $k<p-1$ one can compute $B_{k} / k$ modulo $p$, and then

$$
\frac{B_{k}}{k}\left(x^{k}-1\right) \equiv_{p} x^{k-1} \sum_{j=1}^{p-1} j^{k-1}\left\lfloor\frac{j x}{p}\right\rfloor .
$$

Finally, for an odd prime $p$ and for $a \in\{1, \ldots, p-1\}$, after evaluating the sum of the corresponding geometric series, one has

$$
\begin{gathered}
\sum_{i=1}^{p-1} a^{i} \equiv_{p} \begin{cases}0, & \text { if } a \neq 1, \\
-1, & \text { if } a=1,\end{cases} \\
\sum_{\substack{k=2 \\
2 \mid k}}^{p-3} a^{k-1} \equiv_{p} \begin{cases}-\frac{1}{a}, & \text { if } a^{2} \not_{p} 1, \\
-\frac{3}{2 a}, & \text { if } a^{2} \equiv_{p} 1,\end{cases}
\end{gathered}
$$

where the second sum runs only on the even indices for a prime $p>3$.

\section{Proof of item (1) of Theorem 2}

For $n=1$, the two rings are isomorphic, hence polynomially equivalent. For $p^{n}=4$, by computing the operation tables, it is easy to check that the following polynomials satisfy the requirements:

$$
\begin{aligned}
& f_{1}(x, y)=x+y+2 x y, \\
& g_{1}(x, y)=x y, \\
& f_{2}(x, y)=x+y+t x y, \\
& g_{2}(x, y)=x y .
\end{aligned}
$$

Here, we denoted the additions and the multiplications for both rings in the usual way, because we believe that it does not cause confusion and the formulas are more understandable this way. Similarly, the following polynomials satisfy the requirements for $p^{n}=8$ :

$$
\begin{aligned}
& f_{1}(x, y)=x+y+2 x y+x y(1+x)(1+y)+2 x y\left(1+x^{2}\right)\left(1+y^{2}\right), \\
& g_{1}(x, y)=x y+x^{2} y^{2}(3+x)(3+y) \\
& f_{2}(x, y)=x+y+t x y+x y(1+x)(1+y)+t x y(x+y)^{2}, \\
& g_{2}(x, y)=x y+x^{2} y^{2}(1+x)(1+y) .
\end{aligned}
$$

In the remaining of Section 3, $p$ denotes an odd prime. Let $\mathcal{R}_{1}=\mathbb{Z}_{p^{2}}$, $\mathcal{R}_{2}=\mathbb{Z}_{p}[t] /\left(t^{2}\right)$. Let $P=\{0,1, \ldots, p-1\}$. We identify the elements of $\mathcal{R}_{1}$ and $\mathcal{R}_{2}$ with the elements of $P \times P$ via the bijections $x_{0}+$ $p x_{1} \mapsto\left(x_{0}, x_{1}\right)$ and $x_{0}+t x_{1} \mapsto\left(x_{0}, x_{1}\right)\left(x_{0}, x_{1} \in P\right)$. Thus, we consider 
both $\mathcal{R}_{1}$ and $\mathcal{R}_{2}$ on the domain $P \times P$, i.e. $\mathcal{R}_{1}=\left(P \times P,{ }_{1}, \times_{1}\right)$, $\mathcal{R}_{2}=\left(P \times P,+_{2}, \times_{2}\right)$.

3.1. Addition. Now, we have $\left(x_{0}, x_{1}\right)+_{1}\left(y_{0}, y_{1}\right)=\left(x_{0}+_{p} y_{0}, x_{1}+_{p}\right.$ $\left.y_{1}+{ }_{p} a\left(x_{0}, y_{0}\right)\right)$, where

$$
a\left(x_{0}, y_{0}\right)= \begin{cases}0, & \text { if } x_{0}+y_{0}<p \\ 1, & \text { if } x_{0}+y_{0} \geq p\end{cases}
$$

is the carry-on part of the addition in base $p$. Addition in $\mathcal{R}_{2}$ is the modulo $p$ addition in both coordinates: $\left(x_{0}, x_{1}\right)+_{2}\left(y_{0}, y_{1}\right)=\left(x_{0}+_{p}\right.$ $\left.y_{0}, x_{1}+_{p} y_{1}\right)$. Thus, to express the operation $+_{1}$ in $\mathcal{R}_{2}$, one needs to find a polynomial over $\mathcal{R}_{2}$ (expressed by $+_{2}$ and $\times_{2}$ ) representing $a\left(x_{0}, y_{0}\right)$. Let $f_{2}(x, y)=x+{ }_{2} y+{ }_{2} t \times_{2} A(x, y)$ over $\mathcal{R}_{2}$, where

$$
A(x, y)=\sum_{i=1}^{p-1} \sum_{k=0}^{p-i-1} \frac{1}{p-i}(-1)^{k+i+1} B_{k}\left(\begin{array}{c}
p-i \\
k
\end{array}\right) x^{i} y^{p-i-k},
$$

and every sum uses $+_{2}$, and every multiplication uses $\times_{2}$. Now, $B_{k}$ appears in the formula only for $k<p-1$, and thus can be calculated modulo $p$. The fraction $1 /(p-i)$ can be calculated modulo $p$, as well. Hence, $f_{2}$ is a polynomial over $\mathcal{R}_{2}$. Moreover,

$f_{2}\left(x_{0}+{ }_{2} t \times{ }_{2} x_{1}, y_{0}+{ }_{2} t \times{ }_{2} y_{1}\right)=x_{0}+{ }_{2} y_{0}+{ }_{2} t \times{ }_{2}\left(x_{1}+{ }_{2} y_{1}+{ }_{2} A\left(x_{0}, y_{0}\right)\right)$

over $\mathcal{R}_{2}$, that is,

$$
f_{2}\left(\left(x_{0}, x_{1}\right),\left(y_{0}, y_{1}\right)\right)=\left(x_{0}+{ }_{p} y_{0}, x_{1}+{ }_{p} y_{1}+{ }_{p} A\left(x_{0}, y_{0}\right)\right) .
$$

In the following, we show that $A\left(x_{0}, y_{0}\right)=a\left(x_{0}, y_{0}\right)$, which proves that $+_{1}$ is a polynomial over $\mathcal{R}_{2}$. For better understanding, we drop the index of $x_{0}$ and $y_{0}$ : let $x, y \in P$, we prove $A(x, y)=a(x, y)$. If $y=0$, then $A(x, y)=0=a(x, y)$. Otherwise, $y \in\{1, \ldots, p-1\}$, and

$$
A(x, y) \equiv_{p} \sum_{i=1}^{p-1} x^{i} \frac{1}{p-i} \sum_{k=0}^{p-i-1}(-1)^{k+i+1} B_{k}\left(\begin{array}{c}
p-i \\
k
\end{array}\right) y^{p-i-k}
$$

(we apply (1) with $m=p-i-1$ )

$$
=\sum_{i=1}^{p-1}(-1)^{i+1} x^{i} \sum_{k=1}^{y} k^{p-i-1}
$$

(for $k \in\{1, \ldots, p-1\}$ we have $k^{p-1} \equiv_{p} 1$ )

$$
\equiv_{p} \sum_{k=1}^{y} \sum_{i=1}^{p-1}(-1)^{i+1} x^{i} k^{-i} \equiv_{p}-\sum_{k=1}^{y} \sum_{i=1}^{p-1}\left(-\frac{x}{k}\right)^{i} \text {. }
$$


By (4), we have

$$
\sum_{i=1}^{p-1}\left(-\frac{x}{k}\right)^{i} \equiv_{p} \begin{cases}-1, & \text { if } k \equiv_{p}-x \\ 0, & \text { if } k \not \equiv_{p}-x\end{cases}
$$

Now, there exists at most one $k \in\{1, \ldots, y\}$ such that $k \equiv_{p}-x$, and such a $k$ exists if and only if $y \geq p-x$, that is if $x+y \geq p$. Thus,

$$
-\sum_{k=1}^{y} \sum_{i=1}^{p-1}\left(-\frac{x}{k}\right)^{i} \equiv_{p} \begin{cases}-\sum_{k=1}^{y} 0=0, & \text { if } x+y<p \\ -\sum_{i=1}^{p-1} 1^{i} \equiv_{p} 1, & \text { if } x+y \geq p .\end{cases}
$$

Therefore, $A\left(x_{0}, y_{0}\right)=a\left(x_{0}, y_{0}\right)$ for arbitrary $x_{0}, y_{0} \in P$, and hence the polynomial $f_{2}$ corresponds to the addition of $\mathcal{R}_{1}$. Similarly, the polynomial $f_{1}(x, y)=x+{ }_{1} y-{ }_{1} p \times_{1} A(x, y)$ over $\mathcal{R}_{1}$ expresses the addition of $\mathcal{R}_{2}$.

3.2. Multiplication. We continue with the multiplication in a similar fashion. Now, $\left(x_{0}, x_{1}\right) \times_{1}\left(y_{0}, y_{1}\right)=\left(x_{0} \times_{p} y_{0}, x_{0} \times{ }_{p} y_{1}+_{p} x_{1} \times{ }_{p} y_{0}+_{p}\right.$ $\left.m\left(x_{0}, y_{0}\right)\right)$, where

$$
m\left(x_{0}, y_{0}\right)=\left\lfloor\frac{x_{0} y_{0}}{p}\right\rfloor
$$

is the carry-on part of the multiplication in base $p$. Multiplication in $\mathcal{R}_{2}$ is similar, except there is no carry-on part: $\left(x_{0}, x_{1}\right) \times_{2}\left(y_{0}, y_{1}\right)=$ $\left(x_{0} \times_{p} y_{0}, x_{0} \times_{p} y_{1}+_{p} x_{1} \times_{p} y_{0}\right)$. Thus, to express the operation $\times_{1}$ in $\mathcal{R}_{2}$, one needs to find a polynomial over $\mathcal{R}_{2}$ (expressed by $+_{2}$ and $\times_{2}$ ) representing $m\left(x_{0}, y_{0}\right)$. Let $g_{2}(x, y)=x \times_{2} y+{ }_{2} t \times_{2} M(x, y)$ over $\mathcal{R}_{2}$, where

$$
M(x, y)=\sum_{k=1}^{p-2} \frac{B_{k}}{k}\left(x-x^{p-k}\right)\left(y-y^{p-k}\right),
$$

and every sum uses $+_{2}$, and every multiplication uses $\times_{2}$. Now, $B_{k}$ appears in the formula only for $k<p-1$, and thus can be calculated modulo $p$. The fraction $1 / k$ can be calculated modulo $p$, as well. Hence, $g_{2}$ is a polynomial over $\mathcal{R}_{2}$. Moreover,

$$
\begin{aligned}
& g_{2}\left(x_{0}+{ }_{2} t \times{ }_{2} x_{1}, y_{0}+{ }_{2} t \times \times_{2} y_{1}\right)= \\
& x_{0} \times{ }_{2} y_{0}+{ }_{2} t \times{ }_{2}\left(x_{0} \times{ }_{2} y_{1}+{ }_{2} x_{1} \times{ }_{2} y_{0}+{ }_{2} M\left(x_{0}, y_{0}\right)\right)
\end{aligned}
$$

over $\mathcal{R}_{2}$, that is,

$$
g_{2}\left(\left(x_{0}, x_{1}\right),\left(y_{0}, y_{1}\right)\right)=\left(x_{0} \times_{p} y_{0}, x_{0} \times_{p} y_{1}+{ }_{p} x_{1} \times_{p} y_{0}+{ }_{p} M\left(x_{0}, y_{0}\right)\right) \text {. }
$$

In the following, we show that $M\left(x_{0}, y_{0}\right)=m\left(x_{0}, y_{0}\right)$, which proves that $\times_{1}$ is a polynomial over $\mathcal{R}_{2}$. For better understanding, we drop the index of $x_{0}$ and $y_{0}$ : let $x, y \in P$, we prove $M(x, y)=m(x, y)$.

For $x=0$, or $y \in\{0,1\}$ the equation $M(x, y)=m(x, y)$ is clear. Otherwise, $x^{p-1}=1, y^{p-1}=1$, and the term for the index $k=1$ in $M(x, y)$ is $B_{1} / 1 \cdot\left(x-x^{p-1}\right) \cdot\left(y-y^{p-1}\right)=-(x-1)(y-1) / 2$. For $y=p-1 \equiv_{p}-1$ and for even $k$ we have $y-y^{p-k} \equiv_{p} 0$. Since $B_{k}=0$ 
for odd $k \geq 3$, we have then $M(x, p-1) \equiv_{p}-(x-1) \cdot(-2) / 2=$ $x-1=\lfloor x(p-1) / p\rfloor=m(x, p-1)$. This finishes the proof in case $y \in\{0,1, p-1\}$, and hence the case $p=3$. Assume $x \neq 0, y \notin$ $\{0,1, p-1\}, p>3$. Now,

$$
\sum_{k=1}^{p-2} \frac{B_{k}}{k}\left(x-x^{p-k}\right)\left(y-y^{p-k}\right)
$$

(we cut the sum for $k=1$ and use that if $2 \nmid k \geq 3$, then $B_{k}=0$ )

$$
\equiv_{p}-\frac{(x-1)(y-1)}{2}+\sum_{\substack{k=2 \\ 2 \mid k}}^{p-3} \frac{B_{k}}{k}\left(x-x^{p-k}\right)\left(y-y^{p-k}\right)
$$

(we have $x \equiv_{p} x^{p}$ )

$$
\begin{aligned}
& \equiv_{p}-\frac{(x-1)(y-1)}{2}+\sum_{\substack{k=2 \\
2 \mid k}}^{p-3} \frac{B_{k}}{k}\left(x^{p}-x^{p-k}\right)\left(y-y^{p-k}\right) \\
& \equiv_{p}-\frac{(x-1)(y-1)}{2}+\sum_{\substack{k=2 \\
2 \mid k}}^{p-3} x^{p-k}\left(y-y^{p-k}\right) \cdot \frac{B_{k}}{k}\left(x^{k}-1\right)
\end{aligned}
$$

(we apply (3) for $2 \mid k$ )

$$
\equiv_{p}-\frac{(x-1)(y-1)}{2}+\sum_{\substack{k=2 \\ 2 \mid k}}^{p-3} x^{p-k}\left(y-y^{p-k}\right) \cdot x^{k-1} \sum_{j=1}^{p-1} j^{k-1}\left\lfloor\frac{j x}{p}\right\rfloor
$$

(we have $x^{p-k} \cdot x^{k-1}=x^{p-1} \equiv_{p} 1, y^{p-k}=y^{p-1} \cdot y^{1-k} \equiv_{p} y^{1-k}$ )

$$
\begin{aligned}
& \equiv_{p}-\frac{(x-1)(y-1)}{2}+\sum_{\substack{k=2 \\
2 \mid k}}^{p-3}\left(y-y^{1-k}\right) \sum_{j=1}^{p-1} j^{k-1}\left\lfloor\frac{j x}{p}\right\rfloor \\
(6) \quad & =-\frac{(x-1)(y-1)}{2}+\sum_{j=1}^{p-1}\left\lfloor\frac{j x}{p}\right\rfloor \sum_{\substack{k=2 \\
2 \mid k}}^{p-3}\left(y-y^{1-k}\right) j^{k-1} .
\end{aligned}
$$

By (5), we have

$$
\begin{array}{r}
\sum_{\substack{k=2 \\
2 \mid k}}^{p-3} y j^{k-1}=y \sum_{\substack{k=2 \\
2 \mid k}}^{p-3} j^{k-1} \equiv_{p} \begin{cases}-y / j, & \text { if } j \equiv_{p} \pm 1, \\
-3 y /(2 j), & \text { if } j \equiv_{p} \pm 1,\end{cases} \\
-\sum_{\substack{k=2 \\
2 \mid k}}^{p-3} y^{1-k} j^{k-1}=-\sum_{\substack{k=2 \\
2 \mid k}}^{p-3}\left(\frac{j}{y}\right)^{k-1} \\
\equiv_{p} \begin{cases}y / j, & \text { if } j \equiv_{p} \pm y, \\
3 y /(2 j), & \text { if } j \equiv_{p} \pm y,\end{cases}
\end{array}
$$


and since $y \in\{2, \ldots, p-2\}$, we obtain

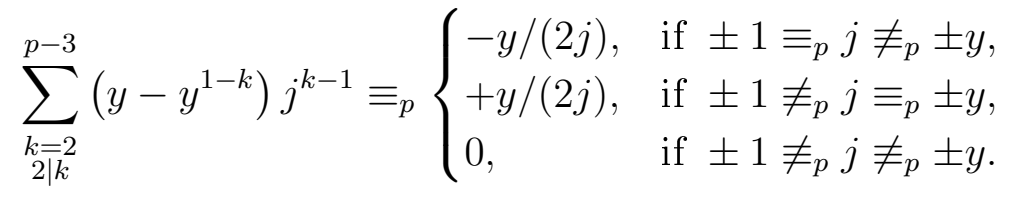

Now, we cut the sum in (6) into five parts: two parts for $\pm 1 \equiv_{p} j \not_{p}$ $\pm y$, two parts for $\pm 1 \not_{p} j \equiv_{p} \pm y$, and one part for $j \notin\{ \pm 1, \pm y\}$ :

$$
\begin{aligned}
& -\frac{(x-1)(y-1)}{2}+\sum_{j=1}^{p-1}\left\lfloor\frac{j x}{p}\right\rfloor \sum_{\substack{k=2 \\
2 \mid k}}^{p-3}\left(y-y^{1-k}\right) j^{k-1} \\
& \equiv_{p}-\frac{(x-1)(y-1)}{2}+\underbrace{\left\lfloor\frac{x}{p}\right\rfloor \cdot \frac{-y}{2}}_{j=1}+\underbrace{\left\lfloor\frac{(p-1) x}{p}\right\rfloor \cdot \frac{y}{2}}_{j=p-1} \\
& +\underbrace{\left\lfloor\frac{y x}{p}\right\rfloor \cdot \frac{1}{2}}_{j=y}+\underbrace{\left\lfloor\frac{(p-y) x}{p}\right\rfloor \cdot \frac{-1}{2}}_{j=p-y}+\sum_{\substack{j=2 \\
j \neq \pm y}}^{p-3}\left\lfloor\frac{j x}{p}\right\rfloor \cdot 0
\end{aligned}
$$

(we have $\lfloor x / p\rfloor=0,\lfloor(p-1) x / p\rfloor=\lfloor x-x / p\rfloor=x-1$, and similarly $-\lfloor(p-y) x / p\rfloor=-\lfloor x-y x / p\rfloor=-(x-1)+\lfloor y x / p\rfloor)$

$$
\begin{aligned}
& \equiv_{p}-\frac{(x-1)(y-1)}{2}+0+\frac{(x-1) y}{2} \\
& +\left\lfloor\frac{y x}{p}\right\rfloor \cdot \frac{1}{2}-\frac{x-1}{2}+\left\lfloor\frac{y x}{p}\right\rfloor \cdot \frac{1}{2}+0=\left\lfloor\frac{y x}{p}\right\rfloor .
\end{aligned}
$$

Therefore, $M\left(x_{0}, y_{0}\right)=m\left(x_{0}, y_{0}\right)$ for arbitrary $x_{0}, y_{0} \in P$, and hence the polynomial $g_{2}$ corresponds to the multiplication of $\mathcal{R}_{1}$. Similarly, the polynomial $g_{1}(x, y)=x+{ }_{1} y-{ }_{1} p \times{ }_{1} M(x, y)$ over $\mathcal{R}_{1}$ expresses the multiplication of $\mathcal{R}_{2}$.

\section{Proof of item (2) of Theorem 2}

Let $\mathcal{R}_{1}=\mathbb{Z}_{p^{n}}, \mathcal{R}_{2}=\mathbb{Z}_{p}[t] /\left(t^{n}\right)$, and assume that they are polynomially equivalent via $\varphi: \mathcal{R}_{1} \rightarrow \mathcal{R}_{2}$. By Lemma 4 we may assume $\varphi\left(0_{1}\right)=0_{2}$. Let $f_{2}$ over $\mathcal{R}_{2}$ correspond to the addition in $\mathcal{R}_{1}$. Let $\mathcal{I}_{1}$ be the unique ideal in $\mathcal{R}_{1}$ containing $p^{2}$-many elements, i.e. $\mathcal{I}_{1}=\left(p^{n-2}\right)$, and let $\mathcal{I}_{2}$ be the unique ideal in $\mathcal{R}_{2}$ containing $p^{2}$-many elements, i.e. $\mathcal{I}_{2}=\left(t^{n-2}\right)$. Then by Lemma 4 we have $\varphi\left(\mathcal{I}_{1}\right)=\mathcal{I}_{2}$. In this section 0 denotes the zero element of $\mathcal{R}_{2},+$ and . denote the addition and multiplication of $\mathcal{R}_{2}$. Assume $n \geq 3$, then $\mathcal{I}_{2}^{3}=(0)$.

Consider $f_{2}(x, y)$ over $\mathcal{R}_{2}$, restricted to $\mathcal{I}_{2}$. This function corresponds to the addition over $\mathcal{R}_{1}$ restricted to $\mathcal{I}_{1}$. Since $\mathcal{I}_{2}^{3}=(0)$, for every $x, y \in \mathcal{I}_{2}$ the function $f_{2}$ attains the same value at $(x, y) \in \mathcal{I}_{2} \times \mathcal{I}_{2}$ as $a+b x+c y+d x y+e x^{2}+f y^{2}$ for some $a, b, c, d, e, f \in \mathcal{R}_{2}$. Now, 
$f_{2}(0,0)=0$ implies $a=0, f_{2}(x, 0)=x$ implies $b x+e x^{2}=x, f_{2}(0, y)=y$ implies $c y+f y^{2}=y$, hence $f_{2}^{\prime}(x, y)=x+y+d x y$ attains the same values on $\mathcal{I}_{2}$ as $f_{2}$. By induction on $m$, it is easy to prove that for every positive integer $m$ we have $f_{2}^{\prime}\left(f_{2}^{\prime}\left(\ldots f_{2}^{\prime}\left(f_{2}^{\prime}(x, x), x\right), \ldots, x\right), x\right)=$ $m x+d\left(\begin{array}{c}m \\ 2\end{array}\right) x^{2}$, if we compose the polynomial $f_{2}^{\prime}$ with itself $m-1$-many times. Consider the case $m=p$. For $p>2$, by $p \mid\left(\begin{array}{l}p \\ 2\end{array}\right)$ we obtain that $f_{2}^{\prime}\left(f_{2}^{\prime}\left(\ldots f_{2}^{\prime}\left(f_{2}^{\prime}(x, x), x\right), \ldots, x\right), x\right)$ is the constant 0 function over $\mathcal{I}_{2}$, while $x+{ }_{1} x+{ }_{1} \cdots+{ }_{1} x=p \times_{1} x$ is not a constant function over $\mathcal{I}_{1}$. This contradiction proves that if $p>2, n \geq 3$, then $\mathcal{R}_{1}$ and $\mathcal{R}_{2}$ are not polynomially equivalent.

If $n \geq 4$, then already $\mathcal{I}_{2}^{2}=(0)$. Thus, $f_{2}^{\prime}(x, y)=x+y$, and $f_{2}^{\prime}\left(f_{2}^{\prime}\left(\ldots f_{2}^{\prime}\left(f_{2}^{\prime}(x, x), x\right), \ldots, x\right), x\right)=m x$. Considering $m=p$, we obtain that $f_{2}^{\prime}\left(f_{2}^{\prime}\left(\ldots f_{2}^{\prime}\left(f_{2}^{\prime}(x, x), x\right), \ldots, x\right), x\right)$ is the constant 0 function over $\mathcal{I}_{2}$, while $x+{ }_{1} x+{ }_{1} \cdots+_{1} x=p \times_{1} x$ is not a constant function over $\mathcal{I}_{1}$. This contradiction proves that if $n \geq 4$, then $\mathcal{R}_{1}$ and $\mathcal{R}_{2}$ are not polynomially equivalent.

\section{Proof of Corollary 3}

Let us use the notations of [3], that is $A=\mathbb{Z}_{p^{2}}, B=p \mathbb{Z}_{p^{3}}, C=p^{2} \mathbb{Z}_{p^{4}}$, $D=\mathbb{Z}_{p} \oplus \mathbb{Z}_{p}, E$ and $F$ are the two noncommutative $p^{2}$-element rings, $G=\mathbb{Z}_{p}[t] /\left(t^{2}\right), H=\mathbb{Z}_{p} \oplus p \mathbb{Z}_{p^{2}}, I=t \mathbb{Z}_{p}[t] /\left(t^{3}\right), J=p \mathbb{Z}_{p^{2}} \oplus p \mathbb{Z}_{p^{2}}$, and $K$ is the $p^{2}$-element field, where $\oplus$ denotes the direct sum of rings.

Now, $A$ and $G$ are polynomially equivalent by Theorem 2 . The proof detailed in Section 4 shows that $B$ and $I$ are not polynomially equivalent for $p \neq 2$. For $p=2, \mathbb{Z}_{8}$ and $\mathbb{Z}_{2}[t] /\left(t^{3}\right)$ are polynomially equivalent by Theorem 2. Moreover, as described in the beginning of Section 3, the polynomials $f_{1}, g_{1}$ over $\mathbb{Z}_{8}$ exist over the unique fourelement ideal $B$, and the polynomials $f_{2}, g_{2}$ over $\mathbb{Z}_{2}[t] /\left(t^{3}\right)$ exist over the unique four-element ideal $I$. Thus $B$ and $I$ are polynomially equivalent for $p=2$. The rings $E$ and $F$ are opposite rings of each other, thus they are polynomially equivalent $\left(x+{ }_{1} y=x+{ }_{2} y, x \times_{1} y=y \times_{2} x\right)$. They cannot be polynomially equivalent to the other rings, as $E$ and $F$ are noncommutative, the other rings are commutative.

Polynomially equivalent rings must have the same ideal structure by Lemma 4, and the factors by the corresponding ideals must be polynomially equivalent. Thus, $K$ is not polynomially equivalent to the others, as that is the only simple ring of $p^{2}$ elements. The only ring having $p+1$ nontrivial ideals is $J$, hence it is not polynomially equivalent to any of the other rings. There are two rings having two nontrivial ideals $(D$ and $H$ ), and in $D$ both ideals are isomorphic to the $p$-element field, while in $H$ one of the ideals is isomorphic to the $p$-element zeroring. Since the factors by the corresponding ideals isomorphic to the $p$-element field are not polynomially equivalent, neither are $D$ and $H$.

A ring $R$ which is not a zero-ring cannot be polynomially equivalent to $C$, because the multiplication of $R$ cannot be expressed as a 
polynomial over $C$. Namely, every polynomial over $C$ is of the form $g(x, y)=a x+b y+c$. Now, if $g$ corresponds to the multiplication, assuming 0 in $R$ corresponds to 0 in $C$, then $g(0,0)=0$ yields $c=0$, $g(x, 0)=0$ yields $a x=0, g(0, y)=0$ yields $b y=0$, hence $g$ is the 0 function.

Finally, the $\operatorname{ring} A$ is not polynomially equivalent to either $B$ or $I$, because the factors by the unique nontrivial ideal are not polynomially equivalent.

\section{ACKNOWLEDGEMENT}

We are grateful to Erhard Aichinger for drawing our attention to this problem. We thank Peter Mayr for discussions on this topic.

\section{REFERENCES}

[1] A. A. Bulatov, 'Polynomial clones containing the Mal'tsev operation of the groups $\mathbb{Z}_{p^{2}}$ and $\mathbb{Z}_{p} \times \mathbb{Z}_{p}$ ', Mult.-Valued Log. (2) 8 (2002), 193-221 Multiplevalued logic in Eastern Europe.

[2] T. Clausen, 'Theorem', Astron. Nachr. 17 (1840), 351-352.

[3] B. Fine, 'Classification of finite rings of order $p^{2}$ ', Math. Mag. (4) 66 (1993), $248-252$.

[4] S. Frisch, 'Polynomial functions on finite commutative rings', in: Advances in commutative ring theory. Proceedings of the 3rd international conference, Fez, Morocco (ed. D. E. Dobbs et al.), volume 205 of Lecture Notes in Pure and Applied Mathematics (Marcel Dekker, New York, 1999) pp. 323-336.

[5] D. Hobby and R. McKenzie, The structure of finite algebras, volume 76 of Contemporary Mathematics (American Mathematical Society, Providence, 1988).

[6] P. M. Idziak and K. Słomczyńska, 'Polynomially rich algebras', J. Pure Appl. Algebra (1) 156 (2001), 33-68.

[7] K. Ireland and M. Rosen, A Classical Introduction to Modern Number Theory (Springer-Verlag, New York, 1982).

[8] M. Istinger and H. K. Kaiser, 'A characterization of polynomially complete algebras', J. Algebra (1) 56 (1979), 103-110.

[9] W. D. Maurer and J. L. Rhodes, 'A property of finite simple non-abelian groups', Proc. Amer. Math. Soci. 16 (1965), 552-554.

[10] L. Rédei and T. Szele, 'Algebraischzahlentheoretische Betrachtungen über Ringe. I', Acta Math. 79 (1947), 291-320.

[11] I. M. Schottenfels, 'Two non-isomorphic simple groups of the same order 20,160', Ann. of Math. (2) (1-4) 1 (1899-1900), 147-152.

[12] Ch. von Staudt, 'Beweis eines Lehrsatzes, die Bernoullischen Zahlen betreffend', J. Reine Angew. Math. 21 (1840), 372-374.

E-mail address: Georg.Grasegger@risc.jku.at

Doctoral Program Computational Mathematics, Research Institute for Symbolic Computation Johannes Kepler University Linz, AltenBerger Strasse 69, 4040 Linz, Austria

E-mail address: ghorvath@science.unideb.hu

Institute of Mathematics, University of Debrecen, Pf. 12, DebreCEN, 4010, Hungary 
Johannes Kepler University Linz, Institute for Algebra, AltenberGer Strasse 69, 4040 LinZ, Austria

E-mail address: Keith.Kearnes@Colorado.EDU

Department of Mathematics, University of Colorado, Boulder CO 80309-0395 